\title{
El control inmunológico: Una alternativa contra garrapatas del ganado bovino
}

\author{
Immunological control: An alternative against cattle ticks
}

\section{Rodolfo Esteban Lagunes- Quintanilla ${ }^{1 *}$, \\ Carlos Ramón Bautista- Garfias" [1] \\ ${ }^{1}$ Centro Nacional de In-} vestigación Disciplinaria en Salud Animal e Inocuidad, Instituto Nacional de Investigaciones Forestales, Agrícolas y Pecuarias. Carretera Federal Cuernavaca-Cuautla

Col. Progreso, CP. 62550. Jiutepec, Morelos, México.

*Autor de correspondencia: rodolfo.lagunes@gmail.com

Artículo de revisión

Recibido: 09 de mayo de 2019 Aceptado: 24 de agosto de 2019

Como citar: LagunesQuintanilla R, Bautista-Garfias CR (2020) El control inmunológico: Una alternativa contra garrapatas del ganado bovino. Ecosistemas y Recursos Agropecuarios 7(1): e2263. DOI: 10.19136/era.a7n1.2263
RESUMEN. El objetivo fue realizar una revisión actualizada del control inmunológico como alternativa para reducir las poblaciones de garrapatas en los bovinos. La historia de las garrapatas de interés veterinario contiene varios pasajes a través del tiempo. Sin duda, la especie más importante en la ganadería es Rhipicephalus microplus, la cual ocasiona pérdidas económicas considerables a la industria pecuaria originando la utilización de diferentes métodos de control para contrarrestar los efectos adversos causados por éste vector. Recientemente, el problema de la resistencia a los ixodicidas ha motivado el interés por el desarrollo de vacunas contra garrapatas, como medida sugerente de control para disminuir las infestaciones en zonas de alta incidencia e indirectamente bloquear la transmisión de patógenos al ganado bovino. En el desarrollo de vacunas contra garrapatas, se han utilizado diferentes criterios y metodologías para identificar proteínas específicas que puedan ser utilizadas como antígenos vacunales. El control inmunológico de garrapatas mediante la utilización de vacunas, es posible y factible, ya que producen una protección en los animales que se refleja en el desarrollo de las garrapatas, reduciendo las poblaciones de las subsiguientes generaciones. Finalmente, la implementación de programas integrados de control basados en la combinación de distintos componentes como las vacunas, contribuirá en un futuro a disminuir los problemas asociados por garrapatas, mejorando la producción y la salud animal de los hatos ganaderos, y a la vez evitar la contaminación del medio ambiente y de los subproductos derivados de los bovinos para el consumo humano.

Palabras clave: Control inmunológico, garrapatas, Rhipicephalus microplus, vacunas.

ABSTRACT. The aim of this paper is to carry out an updated review of the immunological control as a suggestive alternative to reduce tick populations in cattle. Currently, the history of ticks of veterinary interest contains several passages through time. Certainly, the most important species in the livestock field has been Rhipicephalus microplus, which causes considerable economic losses to the livestock industry causing the use of different control methods to neutralize the adverse effects caused by this vector. Recently, acaricide resistance has motivated interest in the development of vaccines against ticks, as a suggestive control measure to reduce infestations in high incidence areas and indirectly block the transmission of pathogens to cattle. In the development of vaccines against ticks, different criteria and methodologies have been used to identify specific proteins that can be used as vaccine antigens. Immunological control of ticks using vaccines is possible and feasible, since they produce protection in animals that is reflected in the development of ticks, reducing populations of subsequent generations. Finally, the implementation of integrated control programs based on the combination of different components such as vaccines, will contribute in the future to reduce the problems associated with ticks, improving the production and animal health of livestock herds, while avoiding the pollution of the environment and by-products derived from bovines for human consumption.

Key words: Immunological control, Rhipicephalus microplus, ticks, vaccines. 


\section{INTRODUCCIÓN}

Las garrapatas son un problema global que se ha incrementado en los últimos años, afectando animales silvestres, domésticos y al humano; su capacidad para adaptarse a la mayoría de los ecosistemas del mundo pone de manifiesto la evolución biológica que han obtenido a través de generaciones. La hematofagia, es su principal característica para ocasionar daño, que se manifiesta mediante la transmisión de diferentes patógenos como protozoarios (Babesia spp., Theileria spp.), bacterias (Anaplasma spp., Erlichia spp., Borrelia spp.), virus (Flavivirus, Nairovirus) y nematodos (Acanthocheilonema) (de la Fuente et al. 2008), los cuales causan enfermedades en diferentes especies, además de mermas transcendentales en animales en producción (Rodríguez-Vivas et al. 2017). En el caso de explotaciones pecuarias, los efectos se reflejan en los problemas sanitarios y pérdidas económicas que ocasionan a la industria ganadera. Los géneros de importancia en este sector son principalmente Rhipicephalus (Boophilus) spp. y Amblyomma spp., las cuales ocasionan pérdidas que se traducen en la disminución de la producción de carne y leche, la elevación de los costos de producción por tratamientos ixodicidas, así como tratamientos contra enfermedades transmitidas por las garrapatas al ganado, como babesiosis y anaplasmosis (Almazán et al. 2018).

El método más utilizado para su control se basa en el uso de productos químicos, los cuales tienen como función romper el ciclo biológico de garrapata con la aplicación de ixodicidas a intervalos específicos mediante baños de aspersión, inmersión, derrame dorsal e inyectables. Por otra parte, el control no químico, se basa en la selección de hospedadores resistentes, introducción de depredadores naturales, rotación, descanso y quema controlada de praderas, tipo de vegetación e inclusive el control biológico (Rodríguez-Vivas et al. 2014). La realidad es que estos métodos de control no han sido suficientes para disminuir las poblaciones de garrapatas presentes en la ganadería bovina por diferentes circunstancias, destacando principalmente el fenómeno conocido como resistencia. Por lo que se requieren nuevas alternativas y estrategias de control donde se incorpore a las vacunas como medida sinérgica para reducir las poblaciones de garrapatas. El control inmunológico basado en el uso de vacunas representa una alternativa promisoria, debido a que se ha logrado disminuir la infestación de artrópodos y a la vez bloquear la transmisión de patógenos a sus hospedadores (de la Fuente 2000, Willadsen 2004). Al respecto, se han identificado diferentes proteínas como posibles candidatos a vacunas; sin embargo, son pocos los antígenos que han sido evaluados en experimentos de inmunización en campo (de la Fuente y Kocan 2003). El antígeno Bm86, es el componente de las vacunas comerciales contra garrapatas. Estas vacunas disminuyen los parámetros reproductivos de las garrapatas, afectando con ello la progenie. No obstante, presenta limitaciones, por lo que no han tenido un éxito rotundo (García-García et al. 1999, Popara et al. 2013).

Actualmente, el estudio de las vacunas contra garrapatas se ha incrementado debido a la utilización de la vacunología inversa, que comprende el uso de técnicas de biología molecular e ingeniería genética para identificar antígenos protectores y desarrollar vacunas recombinantes. Por otro lado, el comienzo de la era post-genómica, revolucionó la forma de poder diseñar y desarrollar antígenos que pueden ser ensayados de manera experimental; de tal forma, que la búsqueda y el diseño de nuevos candidatos vacunales comienza con el análisis in silico del genoma de interés detectando genes específicos con uso potencial en vacunas (Ferreira y Porco 2008). Por lo anterior, el objetivo del presente artículo es realizar una revisión actualizada del control inmunológico como medida sugerente para reducir las poblaciones de garrapatas que afectan a la ganadería bovina.

\section{IMPORTANCIA GLOBAL DE LAS GARRAPATAS EN EL GANADO BOVINO}

La garrapata Rhipicephalus (Boophilus) microplus es la especie que más afecta al ganado bovino, seguida por el género Amblyomma spp; las cuales presentan ciclos de vida diferente, siendo $R$. 
microplus de ciclo monoxeno y Amblyomma de ciclo heteroxeno; generalmente comparten el mismo hospedador y se les observa cohabitando en regiones tropicales y subtropicales del mundo donde existen las condiciones favorables de humedad y temperatura para el desarrollo del ciclo biológico (Maruyama et al. 2010). En México se encuentran establecidas en las zonas del golfo y el pacífico, donde se localiza la mayor población de bovinos del país, aunque esto no es exclusivo y también se encuentran parasitando diversas especies como perros, equinos y animales silvestres (Almazán et al. 2016).

Los efectos causados por las infestaciones de garrapatas $R$. microplus representan uno de los principales problemas que repercuten en la productividad de los bovinos, sin olvidar el papel que juegan en la transmisión de enfermedades como babesiosis (Babesia bovis, Babesia bigemina) y anaplasmosis (Anaplasma marginale) (Almazán et al. 2018). En México, el problema de garrapatas se ha convertido en emergencia sanitaria al limitar la movilización y comercialización de bovinos infestados a zonas libres o en erradicación, afectando también la exportación de ganado en píe a los Estados Unidos; actividad que genera importantes divisas al país. En promedio se estima que cada garrapata hembra repleta es responsable de la pérdida de $1.37 \mathrm{~g}$ en ganado Bos taurus y de $1.18 \mathrm{~g}$ en cruzas de ganado Bos taurus $\mathrm{x}$ Bos indicus (Jonsson 2006). Las pérdidas económicas causadas por garrapatas $R$. microplus están calculadas en México y en el mundo por el orden de los 573 millones (Rodríguez-Vivas et al. 2017) y 2.5 billones de dólares americanos anuales, respectivamente (Lew-Tabor et al. 2014).

El escenario es complejo, debido a que a través de la historia se menciona que el control de garrapatas se basa en productos químicos denominados ixodicidas; los cuales son exitosos cuando se utilizan de forma adecuada, ya que presentan acción rápida sobre las garrapatas que se encuentran sobre los bovinos, pero con nula actividad sobre las larvas que se encuentran en la vegetación. Lo que obliga a realizar tratamientos continuos enfatizando en aspectos importantes como la residualidad y la selección de poblaciones de garrapatas resistentes; sin olvidar, el efecto adverso sobre el medio ambiente y la contaminación de subproductos destinados al consumo humano (Pérez-Cogollo et al. 2010).

La resistencia a los ixodicidas, ha sido definida como el problema principal en el control químico de las garrapatas por varios expertos en el tema (Rodríguez-Vivas et al. 2007, Rosado-Aguilar et al. 2008, Rosario-Cruz et al. 2009, Pérez-Cogollo et al. 2010), ya que reduce la eficacia de los productos químicos a límites inaceptables. Desafortunadamente, hoy en día se han detectado cepas de garrapatas con resistencia múltiple en algunos estados del país (Fernández-Salas et al. 2012), siendo probable que la situación continúe sí mientras el control del ectoparásito, se base principalmente en el uso de ixodicidas. Estas evidencias ponen de manifiesto que las estrategias basadas en el control químico no son la mejor solución, por lo cual, es necesario la búsqueda de nuevas alternativas de control como el inmunológico. Actualmente, el uso de vacunas como método de control es una herramienta que ha arrojado resultados alentadores en diversas regiones del mundo, convirtiéndose en una de las opciones más promisorias (Guerrero et al. 2012). Las ventajas de las vacunas es que pueden tener un efecto de larga duración, no presentan complicaciones de residualidad en animales y en sus derivados ni de contaminación ambiental, hay menor probabilidad de que se desarrolle resistencia hacia ellas y actúan sobre blancos específicos de las garrapatas (de la Fuente y Kocan 2003). No obstante, es necesario comprender que el nivel de infestación depende de las condiciones ambientales y ecológicas de la región donde se desarrollan las garrapatas, sugiriendo que el cambio climático también tiene impacto sobre los hábitats y ciclos biológicos de los ectoparásitos (Kutz et al. 2009). Por tal motivo, se deben implementar programas integrados de control constituidos por al menos dos métodos diferentes a intervalos específicos con la finalidad de reducir de forma gradual el nivel de infestación larval presente en potreros y animales. 


\section{ZCosistemas igopecuARIOS \\ RESPUESTA INMUNITARIA DEL BOVINO CONTRA GARRAPATAS}

La respuesta inmunitaria se ha dividido con objeto de facilitar su estudio en dos tipos: respuesta inmunitaria innata y respuesta inmunitaria adaptativa (adquirida). De tal forma que ambos tipos de respuesta representan dos aspectos de igual importancia en el sistema inmunitario. La primera, actúa de manera inmediata, mientras que a la segunda le toma más tiempo para desarrollarse (Bautista 2016).

La respuesta inmune innata está conformada por células y proteínas que están siempre presentes y listas para movilizarse y combatir a los microorganismos en el sitio de la infección. Los principales componentes del sistema inmunitario innato son: 1) las barreras epiteliales físicas (piel, mucosas), 2) leucocitos fagocitarios (neutrófilos, monocitos, macrófagos), 3) células dendríticas, 4) linfocitos linfoides innatos (ILC1, ILC2 e ILC3) incluyendo linfocitos asesinos naturales (NK) y el inductor de tejido linfoide (LTi) (Bautista 2016), 5) linfocitos T gamma-delta (Bautista 2011) y 6) proteínas plasmáticas circulantes. Por otra parte, la respuesta adaptativa actúa contra patógenos que son capaces de evadir o superar las defensas inmunitarias innatas. Los componentes del sistema inmunitario adaptativo normalmente se encuentran en reposo; sin embargo, cuando son activados estos componentes se adaptan a la presencia de agentes infecciosos por medio de la activación, proliferación y creación de mecanismos potentes para neutralizar o eliminar los microorganismos. Existen dos tipos de respuesta inmunitaria adaptativa: la inmunidad humoral, mediada por anticuerpos producidos por linfocitos B y la inmunidad celular, mediada por linfocitos T. Otro aspecto importante dentro de los mecanismos protectores, es la participación de la respuesta inflamatoria (Bautista 2010), el sistema del complemento (Dempsey et al. 1996), la lectina ligadora de manosa (Eddie et al. 2009), los receptores tipo Toll (TLRs) (Bautista y Mosqueda 2005) y los péptidos antimicrobianos que se denominan péptidos de defensa del hospedador (HDPs) y que desempeñan una función importante en la respuesta inmune innata (Hancock et al. 2016).
Como parte de la respuesta innata, se encuentran las células epiteliales (Aderem y Underhill 1999), células dendríticas (Esterházy et al. 2016), monocitos (Kraaij et al. 2014), macrófagos (Robinson et al. 2010), células cebadas o mastocitos (Urb y Sheppard 2012), neutrófilos (Kumar y Sharma 2010), eosinófilos (Long et al. 2016) y basófilos (Karasuyama et al. 2011). En el caso de la respuesta adaptativa, las células importantes son los linfocitos $T$ y $\mathrm{B}$; entre los primeros se encuentran los linfocitos cooperadores (Th): Th1, Th2, Th9, Th17, Th22, T reguladores (Treg) y linfocitos $T$ citotóxicos, además de los linfocitos B (Bautista 2016). Existen otras células que se han considerado como eslabones de unión entre la respuesta inmune innata y la adaptativa; se trata de los linfocitos NK, Linfocitos NKT, linfocitos $T \gamma \delta$, que en el caso de los bovinos pueden funcionar como Treg, a diferencia de otras especies (Hoek et al. 2009), y los linfocitos innatos (Artis y Spits 2015). Además, deben considerase a las citocinas como moléculas fundamentales de la respuesta inmunitaria (Tisoncik et al. 2012).

Con base en lo anterior, para el desarrollo de vacunas efectivas contra garrapatas del ganado, es indispensable conocer a fondo los mecanismos inmunitarios que operan después de que un bovino es parasitado por garrapatas (Robbertse et al. 2017). Los elementos inmunitarios del bovino que intervienen contra los componentes de la saliva de las garrapatas incluyen la cascada del complemento, células NK (interferón gamma), macrófagos (interferón gamma y óxido nítrico), células cebadas, neutrófilos, células dendríticas (IL-6, TNF- $\alpha$, IL-12p70) y células no inmunitarias como fibroblastos y queratinocitos (Hovious 2009); sin embargo, la saliva de las garrapatas posee la característica de evadir la respuesta inmunitaria inhibiendo o bloqueando la actividad de los elementos inmunitarios antes mencionados (Hovious 2009). Este mecanismo de defensa no es extraño, ya que, de acuerdo a la literatura las garrapatas parasitan a sus hospedadores desde hace 99 millones de años aproximadamente (Peñalver et al. 2017) y, por lo tanto, éstas han desarrollado diversos mecanismos para evitar ser dañadas por la respuesta inmune pro- 
tectora de sus hospedadores. Cabe señalar que la saliva de las garrapatas contiene al menos 81 moléculas diferentes que modulan la respuesta protectora del hospedador (Simo et al. 2017) (Figura 1). Se ha observado que existe una variación en la resistencia a la infestación con garrapatas entre ganado Bos taurus y Bos indicus, sugiriendo que se debe a una mejor respuesta inmunitaria mediada por células $T$, lo cual hace que el ganado de razas cebuínas sea más resistente que el ganado de origen europeo (Jonsson et al. 2014). En estudios recientes, se demostró que los bovinos resistentes a la infestación por garrapatas son capaces de producir anticuerpos dirigidos contra proteínas específicas de la saliva (Garcia et al. 2017).

\section{VACUNAS CONTRA GARRAPATAS}

El origen de las vacunas anti-garrapatas fue aproximadamente a mediados de los años 80's, en donde se realizaron algunos ensayos inmunizando animales con proteínas derivadas de extractos crudos de hembras semirepletas demostrando un efecto protector contra garrapatas. Sin embargo, se necesitaba el asilamiento e identificación de los antígenos responsables que conferían esta protección para ser producidos y obtenidos puramente (Kemp et al. 1986, Willadsen et al. 1988). Posteriormente, se identificaron proteínas de glándulas salivales en garrapatas Dermacentor andersoni las cuales fueron utilizadas para inmunizar cobayos por diferentes vías adicionado con adyuvante y sin adyuvante, logrando inducir una respuesta inmune protectora que se refleja en la disminución del peso y la fertilidad de las garrapatas (Wikel 1981). Más adelante, se identificó una proteína de $20 \mathrm{kDa}$ en glándulas salivales de Amblyomma americanum asociada al cemento, la cual mostró ser la responsable de la protección en cobayos inmunizados cuando fueron infectados con garrapatas (Brown et al. 1984). En garrapatas Haemaphysalis longicornis se caracterizó una proteína de $29 \mathrm{kDa}$ similar al colágeno denominada p29, posiblemente relacionada a la formación de cemento en el sitio de la lesión; la inmunización de conejos con esta proteína recombinante confirió una reducción del
$40 \%$ en la repleción y $56 \%$ en mortalidad de larvas y ninfas (Mulenga et al. 1999).

A finales de los 80's, comenzaron las primeras investigaciones para desarrollar vacunas contra garrapatas Rhipicephalus (Boophilus) spp. de un solo hospedador, propiciando nuevas posibilidades para la identificación de antígenos protectores con fines de inmunización para controlar las infestaciones por garrapatas (Willadsen y Jongejan 1999, de la Fuente et al. 1999, 2000, de Vos et al. 2001). El sitio más afectado de la garrapata utilizando la vacunación con dichas proteínas fue el intestino medio, observándose daños irreversibles tanto en células epiteliales como en membrana basal mediante histopatología; dando como resultado efectos adversos en el metabolismo, sistema digestivo y capacidad reproductiva de la garrapata (Kemp et al. 1986, Willadsen et al. 1988). Con estos antecedentes surgieron nuevas investigaciones hasta lograr la identificación del antígeno responsable de esta protección. La proteína de mayor interés localizada en extractos crudos de garrapatas hembras fue identificada en el intestino medio como una glicoproteína de membrana, purificada y caracterizada con el nombre de Bm86, la cual tenía la capacidad de producir un daño local e irreversible en el intestino; reflejándose en la disminución de garrapatas ingurgitadas, deficiente postura de huevos y afectando la sobrevivencia de la siguiente generación de larvas (Willadsen et al. 1989). De acuerdo con esto, las primeras vacunas utilizadas para el control de garrapatas en el ganado bovino fueron registradas entre 1993 y 1997, con los nombres comerciales TickGard ${ }^{\circledR}$ y Gavac ${ }^{\circledR}$ en Australia y América Latina respectivamente (de la Fuente et al. 2007). En la Tabla 1, se resume los principales hallazgos encontrados con Bm86 para el control de diferentes especies de garrapatas.

Varios ensayos controlados y de campo realizados para evaluar el efecto del antígeno Bm86 contra $R$. microplus, reportan capacidad protectora, afectando el potencial biótico de las garrapatas y disminuyendo la frecuencia de tratamientos ixodicidas así como la transmisión de patógenos (de la Fuente et al. 1999, de Vos et al. 2001, Canales et al. 2009, Almazán et al. 2010). Sin embargo, 


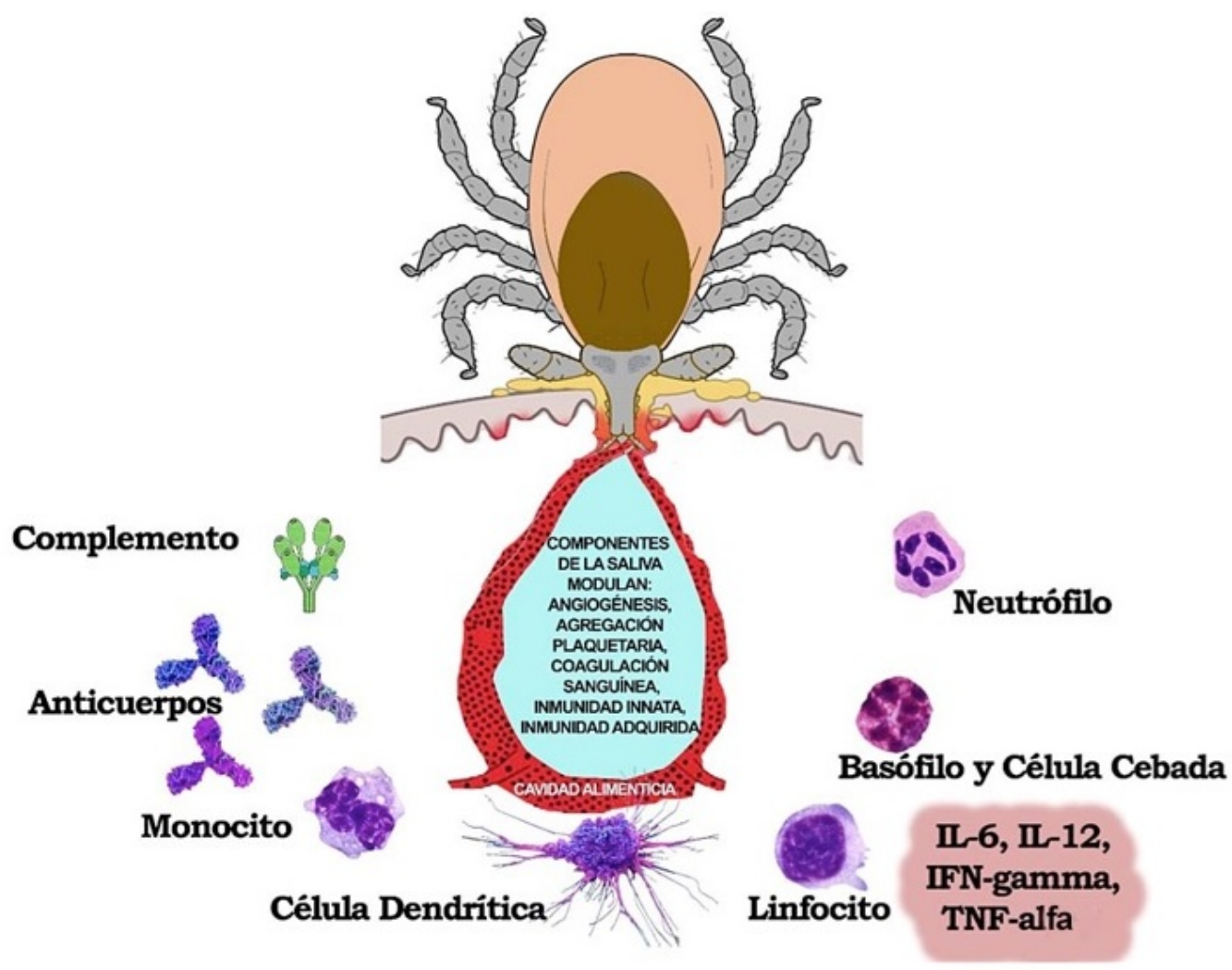

Figura 1. Resumen de la interfase garrapata - respuesta inmunitaria del hospedador. En el esquema se muestran los principales elementos del sistema inmunitario del bovino y los componentes de la saliva de la garrapata que modulan la respuesta inmune protectora del hospedador. IL-6, interleucina 6; IL-12, interleucina 12; IFN, interferón; TNF, factor de necrosis tumoral.

Tabla 1. Eficacia del antígeno Bm86 para el control de diferentes especies de garrapatas en el ganado bovino.

\begin{tabular}{|c|c|c|}
\hline Especie & Eficacia (reducción \%) & Referencia \\
\hline Rhipicephalus annulatus & $100 \%$ & $\begin{array}{l}\text { Fragoso et al. } 1998 \\
\text { Almazán et al. } 2010\end{array}$ \\
\hline $\begin{array}{l}\text { Hyalomma anatolicum } \\
\text { Hyalomma dromedarii }\end{array}$ & $72 \%$ & De Vos et al. 2001 \\
\hline Rhipicephalus decoloratus & $70 \%$ & De Vos et al. 2001 \\
\hline $\begin{array}{l}\text { Amblyomma variegatum } \\
\text { Rhipicephalus appendiculatus }\end{array}$ & $0 \%$ & De Vos et al. 2001 \\
\hline
\end{tabular}

es importante destacar que presenta ciertas limitaciones en la eficacia entre las diferentes cepas de $R$. microplus de acuerdo con su localización geográfica, debido a la presencia de polimorfismos en el gen codificador de la proteína Bm86 (Fragoso et al. 1998, García-García et al. 1999, de Vos et al. 2001). Al respecto se ha demostrado que variaciones superiores al $2.8 \%$ en la secuencia de aminoácidos son suficientes para que el antígeno Bm86 sea ineficaz (García-García et al. 2000). Además, se re- porta que existen poblaciones de $R$. microplus que no presentan reducción en sus parámetros reproductivos después de ser alimentadas en animales inmunizados (García-García et al. 2000, Pruett 2002), lo que confirma la implicación de las variaciones de las secuencias en el locus Bm86 (Peconick et al. 2008) con la adecuada protección inmunológica de la vacunación. Por ello, recientemente en México se desarrolló una vacuna a partir del germoplasma de una cepa mexicana, la cual se registró con el nombre de 

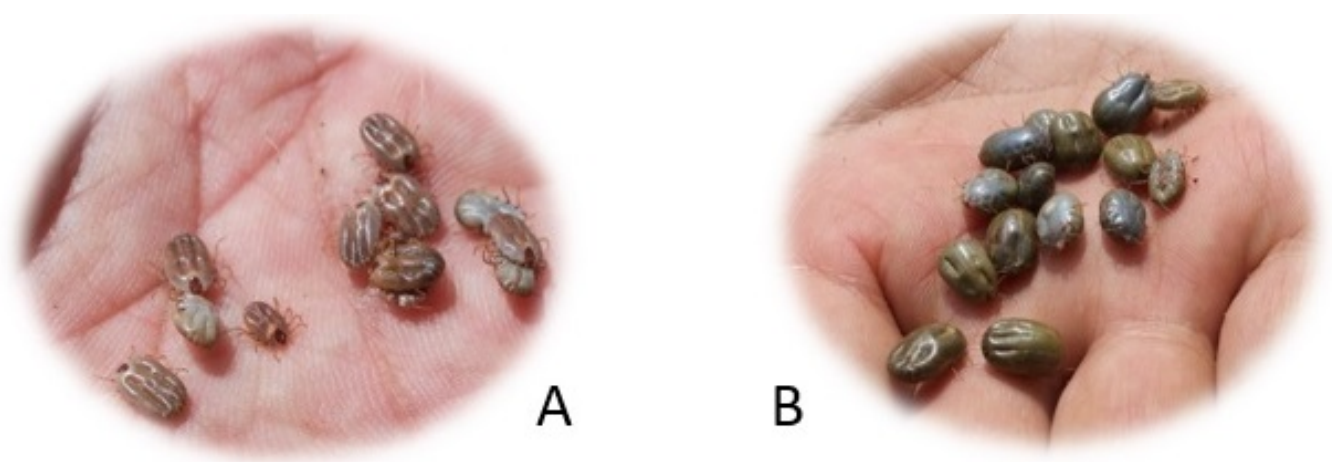

Figura 2. Efecto de la vacuna recombinante Bm86. A) garrapatas alimentadas de bovinos vacunados, B) garrapatas alimentadas de bovinos no vacunados.

Bovimune Ixovac ${ }^{\circledR}$ y se está utilizando en lugares de México donde existen poblaciones de garrapatas multiresistentes con resultados promisorios (Figura 2).

Con base en los resultados alcanzados por el antígeno Bm86, comenzó la investigación de nuevos antígenos, descubriéndose el antígeno Bm91, proteína caracterizada como carboxyldipeptidasa con acción similar a la enzima de la angiotensina, la cual está involucrada en la presión sanguínea y regulación de líquidos en los vertebrados (Jarmey et al. 1995). Los ensayos con esta proteína en combinación con Bm86, muestran eficacia mayor que con los antígenos por separado en contra de $R$. microplus (Willadsen et al. 1996). Posteriormente, se descubrió la proteína Bm95 identificada en células intestinales de la garrapata $R$. microplus al igual que Bm86; sin embargo, ésta proteína se denominó universal, debido a que mostró resultados favorables contra diferentes cepas de garrapatas y de distintas regiones geográficas del mundo (García-García et al. 2000). En un ensayo realizado bajo condiciones controladas utilizando Bm95 en contra de $R$. microplus se obtuvo una eficacia general de la vacunación superior al 80\% (Amit et al. 2009). Además de Bm86 y sus variantes, en las últimas décadas se han caracterizado diferentes antígenos que se encuentran en etapa experimental como candidatos a vacuna; extractos de glándula salival de garrapata (Jittapalapong et al. 2008), Ferritin 2 (Hajdusek et al. 2010), Subolesina (Merino et al. 2013), Aquaporinas (Guerrero et al. 2014), Vitelogenina (Ramírez et al. 2016) entre otros. Estos antígenos han demostrado tener mayor o menor éxito; sin embargo, ninguno de ellos ha alcanzado la fase comercial, y seguramente su efectividad estará sujeta a la variabilidad antigénica de las cepas de garrapatas de donde fueron aisladas (Guerrero et al. 2012).

\section{AVANCES DE LA VACUNOLOGÍA EN GARRAPATAS}

A partir del siglo XXI, el estudio y desarrollo de vacunas ha traído consigo nuevos enfoques en la investigación. Inicialmente, se utilizó al agente infeccioso muerto, atenuado o algún componente purificado del mismo para producir un inmunógeno que protegiera contra el patógeno en cuestión. Posteriormente, se buscó caracterizar la patogénesis de los microorganismos de interés para identificar factores de virulencia con la finalidad de determinar e identificar las principales células de la respuesta inmune y a partir de esto desarrollar vacunas basadas en regiones antigénicas de interés (Ferreira y Porco 2008). Actualmente, el estudio de las vacunas se ha incrementado debido a la utilización de la vacunología inversa, que comprende el uso de técnicas de biología molecular e ingeniería genética para identificar antígenos protectores y desarrollar vacunas recombinantes. Los primeros estudios fueron a partir de bibliotecas de expresión de ADN complementario (cDNA-ELI) en combinación con análisis de secuencias, que en sus inicios la técnica fue propuesta para detectar antígenos protectores contra leishmaniosis (Melby et al. 2000). En 
el caso de garrapatas, se realizaron ensayos de inmunización con bibliotecas de expresión cDNA-ELI a partir de Ixodes scapularis con el fin de identificar y detectar antígenos protectores en ratones infestados artificialmente con larvas de garrapatas $I$. scapularis. Los genes detectados fueron $4 \mathrm{~F} 8$, con homología a nucleotidasa, 4E6 y 4D8 de función desconocida, los cuales fueron utilizados como proteínas recombinantes en un ensayo de inmunización en ovinos, obteniendo una reducción de la infestación de larvas, ninfas y adultos de garrapatas I. scapularis con una eficacia general de 33, 40 y $71 \%$, respectivamente (Almazán et al. 2005). Los estudios subsiguientes condujeron a la caracterización de la proteína 4D8, actualmente conocida como Subolesina, la cual posee una localización intracelular y se encuentra presente en los diferentes estadios de la garrapata y en distintos órganos como intestino, glándulas salivales y ovarios (de la Fuente et al. 2006). El hallazgo fue demostrado mediante la técnica de interferencia de ARN (iARN) en I. scapularis, así como la caracterización de su función en diferentes especies de garrapatas como I. scapularis, A. americanum, Rhipicephalus sanguineus, Dermacentor variabilis, $D$. marginatus (de la Fuente et al. 2005). Donde se observó, que el silenciamiento de este gen produce degeneración de intestinos, glándulas salivales y sistema reproductor de hembras y machos tratados con ARN de doble cadena (ARNdc). Recientemente, la iARN ha sido utilizada como una herramienta de importancia para manipular la expresión génica y caracterizar la función específica del gen en cuestión. En garrapatas fue reportado por primera vez en $A$. americanum introduciendo cadenas cortas de ARNdc para evaluar el silenciamiento de un gen que codificaba para una proteína acoplada a histamina tanto en hembras como en machos (Aljamali et al. 2002). Posteriormente, Nijhof et al. (2007), realizaron un ensayo con el silenciamiento de los genes Bm86, Bm91 y Subolesina en garrapatas $R$. microplus, inyectando ARNdc en hembras adultas y evaluaron el efecto en la oviposición; como resultado observaron daños en la morfología de los huevos ovipositados, infertilidad y anormalidad. Lo que sugiere que estos tres antígenos juegan un papel importante en el desarrollo embrionario de las garrapatas.

Por otro lado, el comienzo de la era postgenómica, revolucionó la forma de poder desarrollar antígenos que pueden ser ensayados de manera experimental; de tal forma, que la búsqueda y el diseño de nuevos candidatos vacunales comienza con el análisis in silico del genoma de interés detectando genes específicos con potencial a vacunas. En el caso concreto de vacunas contra garrapatas se han propuesto diferentes estrategias para diseñar antígenos con características importantes como son: el grado de conservación, presencia de epítopos de células $B$, que estén expuestos a la superficie de la proteína y que sean inmunogénicos. Actualmente, es posible el diseño de antígenos vacunales mediante el uso de programas bioinformáticos, los cuales predicen características antigénicas con diferentes algoritmos para facilitar el desarrollo de un inmunógeno (Crompton et al. 2010). Así fue como Patarroyo et al. (2002), diseñaron tres péptidos sintéticos a partir de la secuencia inferida de Bm86. Se seleccionaron con posibles características antigénicas para que fueran capaces de inducir una respuesta inmune protectora contra garrapatas $R$. microplus cuando eran inoculados en bovinos. De los tres péptidos sintetizados, el que obtuvo una mejor respuesta fue llamado SBm7462, afectando diferentes parámetros reproductivos de las garrapatas (número y peso de hembras repletas, oviposición y eclosión), alcanzando una eficacia general de $81 \%$.

Un estudio similar con el gen subolesina fue realizado por Lagunes et al. (2016), donde analizaron el gen de manera in silico con la finalidad de predecir la región más antigénica de la secuencia previamente publicada en la base de datos del GenBank. Se seleccionó un polipéptido, el cual fue sintetizado de forma recombinante y emulsificado con adyuvante comercial Montanide ${ }^{\circledR}$ para el ensayo de inmunización en bovinos y posterior desafío contra garrapatas $R$. microplus. Los resultados en el número de garrapatas y en el porcentaje de eclosión fueron de 79 y $30 \%$ de reducción, respectivamente; concluyendo que la predicción de la región más antigénica del gen, permite dirigir la respuesta in- 
mune contra los epítopos contenidos en esta parte de la secuencia y de esta manera incrementa la eficacia en el control de las infestaciones por $R$. microplus.

Actualmente, una nueva estrategia que se está implementando para acelerar el proceso de fabricación y evaluación de inmunógenos, es la selección de epítopos específicos de proteínas de superficie que sean conservados y que induzcan una respuesta humoral protectora. Una vez predichos los mejores epítopos dentro de una secuencia aminoacídica se forma un antígeno multiepitópico compuesto de cada uno de los epítopos seleccionados. Los péptidos se sintetizan de manera comercial y la síntesis química se realiza en un sistema de MAPS (Multiple Antigenic Peptide System), el cual consiste en generar los péptidos en forma de tetrámeros u octámeros ramificados para darle mayor inmunogenicidad (Hernández-Silva et al. 2018).

Los avances de la vacunología en garrapatas para caracterizar los genomas de las diferentes especies (Ullmann et al. 2005, Hill et al. 2005), abre nuevas posibilidades en la búsqueda, identificación y descubrimiento de nuevos antígenos protectores contra garrapatas que sean propuestos como candidatos vacunales y que puedan contribuir a controlar múltiples especies de garrapatas e indirectamente reducir la transmisión de agentes patógenos (de la Fuente et al. 2006). Al día de hoy, no se cuenta con vacunas alternativas contra diferentes especies de garrapatas que sean más efectivas que la derivada del antígeno Bm86. Sin embargo, se ha sugerido que la eficacia de las vacunas podría aumentar con la sinergia de otros antígenos o con la utilización de adyuvantes que generen una respuesta inmune más específica. Recientemente, se reportó una nueva modalidad de vacunación contra garrapatas que consiste en la aplicación oral de Subolesina de R. microplus en combinación con Mycobacterium bovis inactivado por calor, procedimiento que demostró una eficacia de $65 \%$ en el control de R. microplus (Con- treras et al. 2019). Es importante señalar que los resultados experimentales obtenidos hasta ahora por las vacunas multiantigénicas han sido escasos y poco satisfactorios, debido a las dificultades para llevar a cabo las formulaciones de los antígenos (cocktails), obteniendo niveles moderados de eficacia en comparación con ciertos antígenos que son aplicados individualmente, posiblemente debido a un efecto antagónico que no permite una protección eficaz; por lo cual, se requieren mayores estudios para lograr la sinergia esperada (Willadsen 2008, Olds et al. 2016).

\section{CONCLUSIONES}

Las investigaciones referentes al control inmunológico de garrapatas han dado un giro durante los últimos años; la biología molecular, genómica funcional, proteómica, interactómica, metabolómica, entre otros, en combinación con la bioinformática, han sido herramientas ampliamente utilizadas para la identificación y caracterización de genes con la finalidad de diseñar inmunógenos con potencial vacunal. Además, el estudio de polimorfismos genéticos y variantes antigénicas de proteínas de interés en distintas zonas del país, favorecerá el desarrollo de vacunas basadas en antígenos locales estableciendo un sistema de control regional de garrapatas. Asimismo, es concebible proponer el uso de nuevas vacunas contra garrapatas basadas en combinación de antígenos sintéticos con adyuvantes apropiados para potenciar una respuesta inmunitaria en los bovinos. Finalmente, aparte de las investigaciones en curso, se requiere implementar programas integrados de control basados en la combinación de los componentes que se tienen a la fecha, con el propósito de reducir las poblaciones de garrapatas en los bovinos y en los potreros, disminuyendo el uso de ixodicidas, mitigando la resistencia y alargando la vida útil de los productos químicos disponibles en el mercado.

\section{LITERATURA CITADA}

Aderem A, Underhill DM (1999) Mechanisms of phagocytosis in macrophages. Annual Review Immunology 17: 593-623. 
Aljamali MN, Sauer JR, Essenberg RC (2002) RNA interference applicability in tick research. Experimental Applied Acarology 28: 89-96.

Almazán C, Kocan KM, Blouin EF, de la Fuente J (2005) Vaccination with recombinant tick antigens for the control of Ixodes scapularis adult infestations. Vaccine 23: 5294-5308.

Almazán C, Lagunes R, Villar M, Canales M, Rosario-Cruz R, Jongejan F, de la Fuente J (2010) Identification and characterization of Rhipicephalus (Boophilus) microplus candidate protective antigens for the control of cattle tick infestations. Parasitology Research 106: 471-479.

Almazán C, Torres-Torres A, Torres-Rodríguez L, Soberanes-Céspedes N, Martín Ortiz-Estrada M (2016) Aspectos biológicos de Amblyomma mixtum (Koch, 1844) en el noreste de México. Quehacer Científico en Chiapas 11: 10-19.

Almazán C, Aguilar-Tipacamu G, Rodríguez S, Mosqueda J, Pérez de León AA (2018) Immunological control of ticks and tick-borne diseases that impact cattle health and production. Frontiers in Bioscience 23: 15351551.

Amit K, Garg R, Yadav CL, Vatsya S, Kumar RR, Sugumar P, Chandran D, Mangamoorib LN, Bedarkar SN (2009) Immune responses against recombinant tick antigen, Bm95, for the control of Rhipicephalus (Boophilus) microplus ticks in cattle. Veterinary Parasitology 165: 119-124.

Artis D, Spits H (2015) The biology of innate lymphoid cells. Nature 517: 293-301.

Bautista GCR, Mosqueda GJJ (2005) Papel de los receptores Toll en la inmunidad innata y su implicación en medicina veterinaria. Veterinaria México 36: 453-468.

Bautista GCR (2010) Inflamación. En: Gutiérrez-Pabello JA (ed). Inmunología veterinaria. El manual Moderno. México. pp: 53-64.

Bautista GCR (2011) Importancia de los linfocitos $T \gamma \delta$ en la respuesta inmunitaria de los bovinos. Veterinaria México 42: 65-75.

Bautista GCR (2016) Respuesta inmunitaria de los bovinos y su papel en la babesiosis. Libro Científico No. 3. INIFAP. Ciudad de México, México. 101p.

Brown SJ, Shapiro SZ, Askenase PW (1984) Characterization of tick antigens inducing host immune resistance. Immunization of Guinea pigs with Amblyomma americanum-derived salivary gland extracts and identification of an important salivary gland protein antigen with Guinea pig anti-tick antibodies. Journal of Immunology 133: 3319-3325.

Canales M, Almazán C, Naranjo V, Jongejan F, de la Fuente J (2009) Vaccination with recombinant Boophilus annulatus Bm86 ortholog protein, Ba86, protects cattle against $B$. annulatus and $B$. microplus infestations. BMC Biotechnology 9. Article number 29. Doi: 10.1186/1472-6750-9-29.

Contreras M, Kasaija PD, Merino O, de la Cruz-Hernandez N, Gortazar C, de la Fuente J (2019) Oral vaccination with a formulation combining Rhipicephalus microplus Subolesin with heat inactivated Mycobacterium bovis reduces tick infestations in cattle. Frontiers in Cellular and Infection Microbiology 9. Article number 45. Doi: 10.3389/fcimb.2019.00045.

Crompton D, Kayala A, Traore B, Kayentao K, Ongoiba A, Weiss GE, Molina DM, Burk ChR, Waisberg M, Jasinskas A, Tan X, Doumbo S, Doumtabe D, Kone Y, Narum DL, Liang X, Doumbo OK, Miller LH, Doolan DL, Baldi P, Felgner PL, Piercea SK (2010) A prospective analysis of the Ab response to Plasmodium falciparum before and after a malaria season by protein microarray. Proceedings of the National Academy of Sciences of the United States of America 107: 6958-6963. 
Dempsey PW, Allison MED, Akkaraju S, Goodnow CC, Fearon DT (1996) C3d of complement as a molecular adjuvant: bridging innate and acquired immunity. Science 271: 348-350.

de la Fuente J, Rodríguez M, Montero C, Redondo M, García-García JC, Méndez L, Serrano E, Valdés M, Enríquez A, Canales M, Ramos E, Boué O, Machado H, Lleonart R (1999) Vaccination against ticks (Boophilus spp.): the experience with the Bm86-based vaccine Gavac ${ }^{\mathrm{TM}}$. Genetic Analysis Biomolecular Engineering 15: $143-148$.

de la Fuente J (2000) Immunological control of ticks through vaccination with Boophilus microplus gut antigens. Annals of the New York Academy of Sciences 916: 617-621.

de la Fuente J, Kocan KM (2003) Advances in the identification and characterization of protective antigens for recombinant vaccines against tick infestations. Vaccine 4: 583-593.

de la Fuente J, Almazán C, Blouin EF, Naranjo V, Kocan KM (2005) RNA interference screening in ticks for identification of protective antigens. Parasitology Research 96: 137-141.

de la Fuente J, Almazán C, Blas-Machado U, Naranjo V, Mangold AJ, Blouin EF, Gortazar C, Kocan KM (2006) The tick protective antigen, 4D8, is a conserved protein involved in modulation of tick blood digestion and reproduction. Vaccine 24: 4082-4095.

de la Fuente J, Almazán C, Canales M, Pérez de la Lastra JM, Kocan KM, Willadsen P (2007) A ten-year review of commercial vaccine performance for control of tick infestations on cattle. Animal Health Research Reviews 8: 23-28.

de la Fuente J, Estrada-Pena A, Venzal JM, Kocan KM, Sonenshine DE (2008) Overview: Ticks as vectors of pathogens that cause disease in humans and animals. Frontiers in Bioscience 13: 6938-6946.

de Vos S, Zeinstra L, Taoufik O, Willadsen P, Jongejan F (2001) Evidence for the utility of the Bm86 antigen from Boophilus microplus in vaccination against other tick species. Experimental Applied Acarology 25: 245-261.

Eddie WK, Takahashi K, Ezekowitz RA, Stuart LM (2009) Mannose-binding lectin and innate immunity. Immunology Reviews 230: 9-21.

Esterházy D, Loschko J, London M, Jove V, Oliveira TY, Mucida D (2016) Classical dendritic cells are required for dietary antigen-mediated induction of peripheral Treg cells and tolerance. Nature Immunology 17: 545-555.

Ferreira J, Porco A (2008) Vacunas derivadas del análisis de los genomas: vacunología inversa. Revista de Ciencia y Tecnología de América 5: 353-358.

Fernández-Salas A, Rodríguez-Vivas RI, Alonso-Díaz MA (2012) First report of a Rhipicephalus microplus tick population multi-resistant to acaricides and ivermectin in the Mexican tropics. Veterinary Parasitology 183: 338-342.

Fragoso H, Hoshman-Rad P, Ortiz M, Rodríguez M, Redondo M, Herrera L, de la Fuente J (1998) Protection against Boophilus annulatus infestations in cattle vaccinated with the $B$. microplus Bm86-containing vaccine Gavac. Vaccine 16: 1990-1992.

Garcia GR, Maruyama SR, Nelson KT, Ribeiro JM, Gardinassi LG, Maia AA, Ferreira BR, Kooyman FN, de Miranda Santos IK (2017) Immune recognition of salivary proteins from the cattle tick Rhipicephalus microplus differs according to the genotype of the bovine host. Parasites \& Vectors 10. Article number 144. Doi: 10.1186/s13071-017-2077-9.

García-García JC, Gonzalez IL, Gonzalez DM, Valdés M, Méndez L, Lamberti J, D’Agostino B, Citroni D, Fragoso H, Ortiz M, Rodríguez M, de la Fuente J (1999) Sequence variations in the Boophilus microplus Bm86 
locus implications for immunoprotection in cattle vaccinated with this antigen. Experimental and Applied Acarology 23: 883-895.

García-García JC, Montero C, Redondo M, Vargas M, Canales M, Boue O, Rodríguez M, Joglar M, Machado H, González IL, Valdés M, Méndez L, de la Fuente J (2000) Control of ticks resistant to immunization with Bm86 in cattle vaccinated with the recombinant antigen Bm95 isolated from the cattle tick Boophilus microplus. Vaccine 18: 2275-2287.

Guerrero F, Miller R, Pérez de León A (2012) Many candidate antigens, but will a commercially viable product emerge? International Journal for Parasitology 42: 421-427.

Guerrero F, Andreotti R, Bendele K, Cunha R, Miller R, Yeater K, Pérez de LA (2014) Rhipicephalus (Boophilus) microplus aquaporin as an effective vaccine antigen to protect against cattle tick infestations. Parasites \& Vectors 7. Article number 475. Doi: 10.1186/s13071-014-0475-9.

Hadjusek O, Almazán C, Loosova G, Villar M, Canales M, Grubhoffer L, Kopacek P, de la Fuente J (2010) Characterization of ferritin 2 for the control of tick infestations. Vaccine 28: 2993-2998.

Hancock REW, Haney EF, Gill EE (2016) The immunology of host defence peptides: beyond antimicrobial activity. Nature Reviews Immunology 16: 321-334.

Hernández-Silva D, Valdez-Espinoza U, Mercado UMA, Aguilar-Tipacamú G, Ramos-Aragón JA, HernándezOrtiz R, Ueti M, Mosqueda J (2018) Immunomolecular characterization of MIC-1, a novel antigen in $B$. bigemina, which contains conserved and immunodominant $\mathrm{B}$ epitopes that induce neutralizing antibodies. Veterinary sciences 5(2): 32. Doi: 10.3390/vetsci5020032.

Hill CA, Wikel SK (2005) The Ixodes scapularis Genome Project: an opportunity for advancing tick research. Trends in Parasitology 21: 151-153.

Hoek A, Rutten VPMG, Kool J, Arkesteijn GJA, Bouwstra RJ, Rhijn IV, Koets AP (2009) Subpopulations of bovine $\mathrm{WC} 1^{+} \gamma \delta \mathrm{T}$ cells rather than $\mathrm{CD} 4^{+} \mathrm{CD} 25^{\text {high }} \mathrm{Foxp}^{+}{ }^{+} \mathrm{T}$ cells act as immune regulatory cell ex vivo. Veterinary Research 40(1): 06. Doi: 10.1051/vetres:2008044.

Hovious JWR (2009) Tick saliva assists the causative agent of Lyme disease in evading host skin's innate immune response. Journal of Investigative Dermatology 129: 2337-2339.

Jarmey JM, Riding GA, Pearson RD, Mckena RV, Willadsen P (1995) Carboxydipeptidase from Boophilus microplus: a "Concealed" antigen whit similarity angiotensin-converting enzime. Insect Biochemistry and Molecular Biology 25: 969-974.

Jittapalapong S, Phichitrasilp T, Chanphao H, Rerkamnuychoke W, Stich R (2008) Immunization with tick salivary gland extracts. Annals of the New York Academy of Sciences 1149: 200-204.

Jonsson NN (2006) The productivity effects of cattle tick (Boophilus microplus) infestation on cattle, with particular reference to Bos indicus cattle and their crosses. Veterinary Parasitology 15: 1-10.

Jonsson NN, Piper EK, Constantinoiu CC (2014) Host resistance in cattle to infestation with the cattle tick Rhipicephalus microplus. Parasite Immunology 36: 553-559.

Karasuyama H, Mukai K, Obata K, Tsujimura Y, Wada T (2011) Nonredundant roles of basophils in immunity. Annual Review of Immunology 29: 45-69.

Kemp DH, Agbede RIS, Johnston LAY, Gough GM (1986) Immunization of cattle against Boophilus microplus using extracts derived from adult female ticks: Feeding and survival of the parasite on the vaccinated cattle. International Journal for Parasitology 16: 115-120. 
Kraaij MD, Vereyken EJ, Leenen PJ, van der Bosch TP, Rezaee F, Betjes MG, Baan CC, Rowshani AT (2014) Human monocytes produce interferon-gamma upon stimulation with LPS. Cytokine 67: 7-12.

Kumar V, Sharma A (2010) Neutrophils: Cinderella of innate immune system. International Immunopharmacology 10: $1325-1334$.

Kutz SJ, Jenkins EJ, Veitch AM, Ducrocq J, Polley L, Elkin B, Lair S (2009) The arctic as a model for anticipating, preventing, and mitigating climate change impacts on host-parasite interactions. Veterinary Parasitology 163: 217-28.

Lagunes R, Domínguez D, Quiroz H, Martínez M, Rosario R (2016) Potential effects on Rhipicephalus microplus tick larvae fed on calves immunized with a Subolesin peptide predicted by epitope analysis. Tropical Biomedicine 33: 726-738.

Lew-Tabor AE, Bruyeres AG, Zhang B, Valle MR (2014) Rhipicephalus (Boophilus) microplus tick in vitro feeding methods for functional (dsRNA) and vaccine candidate (antibody) screening. Ticks and Tick-borne Diseases 5: 500-510.

Long H, Liao W, Wang L, Lu Q (2016) A player and coordinator: the versatile roles of eosinophils in the immune system. Transfusion Medicine and Hemotherapy 43: 96-108.

Maruyama SR, Anatriello E, Anderson JM, Ribeiro JM, Brandao LG, Valenzuela JG, Ferreira BR, Garcia GR, Szabó MP, Patel S, Bishop R, de Miranda-Santos IK (2010) The expression of genes coding for distinct types of glycine-rich proteins varies according to the biology of three metastriate ticks, Rhipicephalus (Boophilus) microplus, Rhipicephalus sanguineus and Amblyomma cajennense. BMC Genomics 11. Article number 363. Doi: 10.1186/1471-2164-11-363.

Melby PC, Ogden GB, Flores HA, Zhao W, Geldmacher C, Biediger NM, Ahuja SK, Uranga J, Melendez M (2000) Identification of vaccine candidates for experimental visceral leishmaniasis by immunization with sequential fractions of a cDNA expression library. Infection and Immunity 68: 5595-602.

Merino O, Antunes S, Mosqueda J, Moreno-Cid JA, Pérez de la Lastra JM, Rosario-Cruz R, Rodríguez S, Domingos A, de la Fuente $J(2013)$ Vaccination with proteins involved in tick-pathogen interactions reduces vector infestations and pathogen infection. Vaccine 31: 5889-5896.

Mulenga A, Sugimoto C, Sako Y, Ohashi K, Musoke A, Shubash M, Onuma M (1999) Molecular characterization of a Haemaphysalis longicornis tick salivary gland-associated 29-kilodalton protein and its effect as a vaccine against tick infestation in rabbits. Journal of Microbiology, Immunology and Infection 67: 1652-1658.

Nijhof AM, Taoufik A, de la Fuente J, Kocan KM, de Vries E, Jongejan F (2007) Gene silencing of the tick protective antigens, Bm86, Bm91 and subolesin, in the one-host tick Boophilus microplus by RNA interference. International Journal for Parasitology 37: 653-662.

Olds CL, Mwaura S, Odongo DO, Scoles GA, Bishop R, Daubenberger C (2016) Induction of humoral immune response to multiple recombinant Rhipicephalus appendiculatus antigens and their effect on tick feeding success and pathogen transmission. Parasites \& Vectors 9. Article number 484. Doi: 10.1186/s13071-0161774-0.

Patarroyo H, Portela W, De Castro O, Pimentel C, Guzmán F, Patarroyo E, Vargas MI, Prates AA, Mendes MA (2002) Immunization of cattle with synthetic peptides derived from the Boophilus microplus gut protein (Bm86). Veterinary Immunology and Immunopathology 88: 163-172.

Peconick A, Sossai S, Girào F, Rodriguez M, Souza e Silva C, Guzman Q, Patarroyo VAM, Vargas MI, Patarroyo $\mathrm{JH}$ (2008) Synthetic vaccine (SBm7462) against the cattle tick Rhipicephalus (Boophilus) microplus: Preser- 
vation of immunogenic determinants in different strains from South America. Experimental Parasitology 119: 37-43.

Peñalver E, Arillo A, Delclòs X, Peris D, Grimaldi DA, Anderson SR, Nascimbene PC, Pérez-de la Fuente R (2017) Ticks parasitised feathered dinosaurs as revealed by Cretaceous amber assemblages. Nature Communications 8. Article number 1924. Doi: 10.1038/s41467-017-01550-z.

Pérez-Cogollo LC, Rodríguez-Vivas RI, Ramirez-Cruz GT, Miller RJ (2010) First report of the cattle tick Rhipicephalus microplus resistant to ivermectin in Mexico. Veterinary Parasitology 168: 165-169.

Popara M, Villar M, Mateos-Hernández L, Fernández de Mera IG, Marina A, del Valle M, Almazán C, Domingos A, de la Fuente $J(2013)$ Lesser protein degradation machinery correlates with higher BM86 tick vaccine efficacy in Rhipicephalus annulatus when compared to Rhipicephalus microplus. Vaccine 31: 4728-4735.

Pruett J (2002) Immunological intervention for the control of ectoparasites of livestock - A review. Journal of Veterinary Parasitology 16: 1-10.

Ramírez R, Rosario R, Domínguez D, Hernández R, Lagunes R, Ortuño D, González CC, Gutiérrez OA, Herrera RSE, Vallejo CA, Martínez VM (2016) Identification of immunogenic proteins from ovarian tissue and recognized in larval extracts of Rhipicephalus (Boophilus) microplus, through an immunoproteomic approach. Experimental Parasitology 170: 227-235.

Robbertse L, Richards SA, Maritz-Olivier C (2017) Bovine immune factors underlying tick resistance: Integration and future directions. Frontiers in Cellular and Infection Microbiololgy 7: 522.

Robinson CM, O'Dee D, Hamilton T, Nau GJ (2010) Cytokines involved in Interferon $\gamma$ production by human macrophages. Journal of Innate Immunology 2: 56-65.

Rodríguez-Vivas RI, Rivas AL, Chowell G, Fragoso SH, Rosario-Cruz R, García Z, Smith SD, Williams JJ, Schwager SJ (2007) Spatial distribution of acaricide profiles (Boophilus microplus strains susceptible or resistant to acaricides) in southeastern Mexico. Veterinary Parasitology 146: 158-169.

Rodríguez-Vivas RI, Rosado-Aguilar JA, Ojeda-Chi MM, Pérez-Cogollo LC, Trinidad-Martínez I, Bolio-González ME (2014) Control integrado de garrapatas en la ganadería bovina. Ecosistemas y Recursos Agropecuarios 1: 295-308.

Rodríguez-Vivas R, Grisi L, Pérez de León A, Humberto Silva H, Torres-Acosta J, Fragoso H, Romero SD, Rosario CR, Saldiernah F, García CD (2017) Potential economic impact assessment for cattle parasites in Mexico. Review. Revista Mexicana de Ciencias Pecuarias 8: 61-74.

Rosado-Aguilar JA, Rodriguez-Vivas RI, Garcia-Vazquez Z, Fragoso-Sanchez H, Ortiz-Nájera A, Rosario-Cruz R (2008) Development of amitraz resistance in field populations of Boophilus microplus (Acari: Ixodidae) undergoing typical amitraz exposure in the Mexican tropics. Veterinary Parasitology 152: 349-353.

Rosario-Cruz R, Almazan C, Miller RJ, Domínguez-García DI, Hernandez-Ortiz R, de la Fuente J (2009) Genetic basis and impact of tick acaricide resistance. Frontiers in Bioscience 14: 2657-2665.

Simo L, Kazimirova M, Richardson J, Bonnet SI (2017) The essential role of tick salivary glands and saliva in tick feeding and pathogen transmission. Frontiers in Cellular and Infection Microbiology 7: 281. Doi: 10.3389/fcimb.2017.00281.

Tisoncik JR, Korth MJ, Simmons CP, Farrar J, Martin TR, Katze MG (2012) Into the eye of the cytokine storm. Microbiology and Molecular Biology Reviews 76: 16-32. 
Ullmann AJ, Lima CM, Guerrero FD, Piesman J, Black WC (2005) Genome size and organization in the blacklegged tick, Ixodes scapularis and the Southern cattle tick, Boophilus microplus. Insect Molecular Biology 14: 217-22.

Urb M, Sheppard DC (2012) The role of mast cells in the defence against pathogens. PLOS Pathogens 8(4): e1002619. Doi 10.1371/journal.ppat.1002619.

Wikel SK (1981) The induction of host resistance to tick infestation with a salivary gland antigen. The American Journal of Tropical Medicine and Hygiene 31: 284-288.

Willadsen P, Mckenna RV, Riding GA (1988) Isolation from the cattle tick Boophilus microplus, of antigenic material capable of eliciting a protective immunological response in the bovine host. International Journal for Parasitology 18: 183-189.

Willadsen P, Riding GA, McKenna RV, Kemp DH, Tellam RL, Nielsen JN, Lahnstein J, Cobon GS, Gough JM (1989) Immunological control of a parasitic arthropod: identification of a protective antigen from Boophilus microplus. Journal of Immunology 143: 1346-1351.

Willadsen P, Smith D, Cobon G, Mckenna RB (1996) Comparative vaccination of cattle against Boophilus microplus whit recombinant antigen Bm86 alone or in combination whit recombinant Bm91. Parasite Immunology 18: 241-246.

Willadsen P, Jongejan F (1999) Immunology of the tick-host interaction and the control of ticks and tick-borne diseases. Parasitology Today 15: 258-262.

Willadsen P (2004) Anti-tick vaccines. Parasitology 129: S367-S387.

Willadsen P (2008) Antigen cocktails: Valid hypothesis or unsubstantiated hope? Trends in Parasitology 24: 164-167. 